\title{
Population Pharmacokinetics Modeling of Levofloxacin in Rabbit After Intravenous Bolus Injection and Peroral Administration
}

\author{
Akhmad Kharis Nugroho' ${ }^{*}$, Puspa Dwi Pratiwi1, Shesanthi Citrariana1, Endang Lukitaningsih ${ }^{2}$, \\ Lukman Hakim $^{3}$
}

1. Department of Pharmaceutics, Faculty of Pharmacy, Universitas Gadjah Mada, Yogyakarta 55281, Indonesia

2. Department of Pharmaceutical Chemistry, Faculty of Pharmacy, Universitas Gadjah Mada, Yogyakarta 55281, Indonesia

3. Department of Pharmacology and Clinical Pharmacy, Faculty of Pharmacy, Universitas Gadjah Mada, Yogyakarta 55281, Indonesia

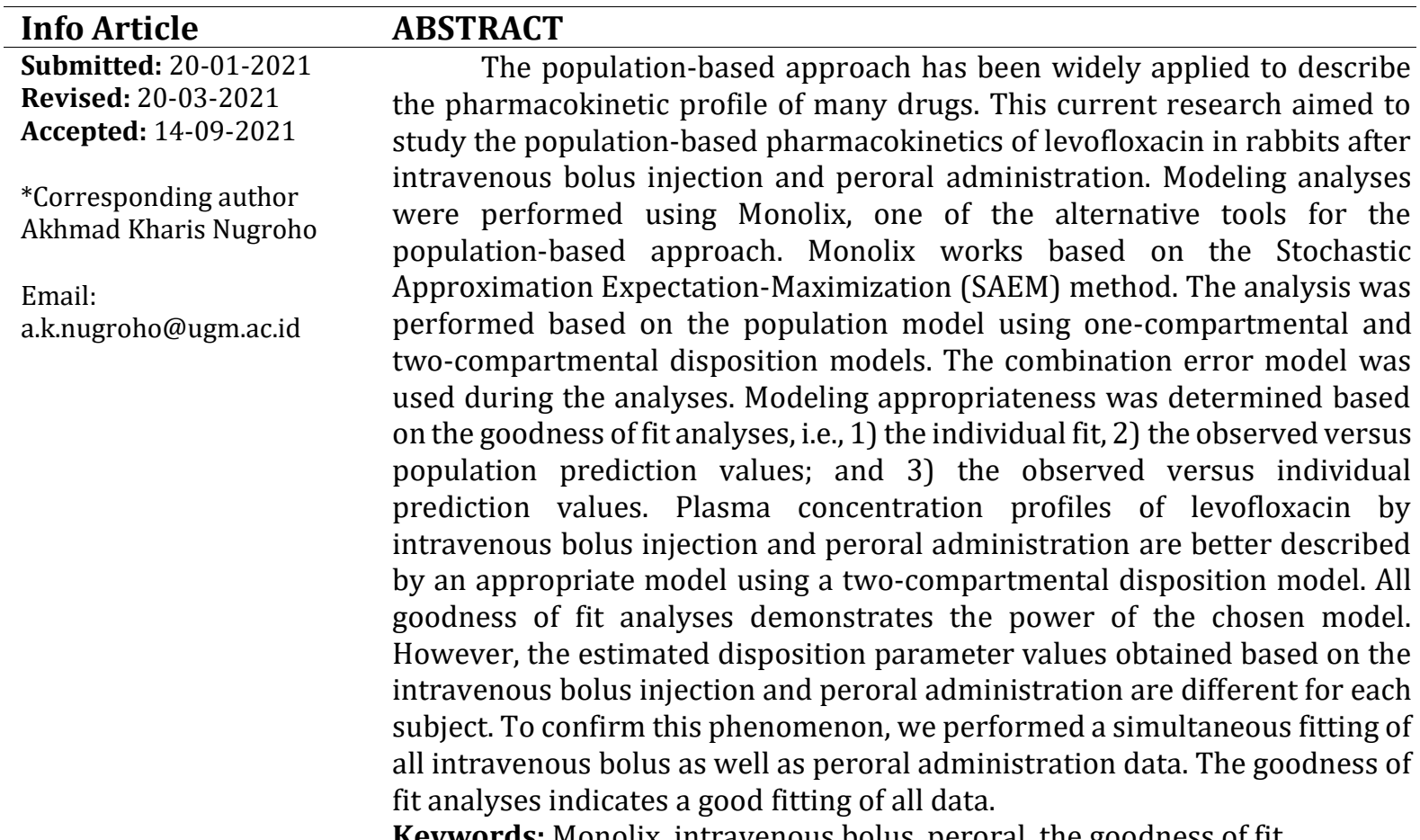

Keywords: Monolix, intravenous bolus, peroral, the goodness of fit

\section{INTRODUCTION}

Levofloxacin is a broad-spectrum fluoroquinolone antibiotic, plays an essential role in the treatment of microbial infections. It has been known that this compound is more active than previous fluoroquinolone generations, i.e., ciprofloxacin (Fu et al., 1992). Levofloxacin is reported to be effective against pneumococci, with greater efficacy than ciprofloxacin. This antibiotic is also used to treat bacterial infections of the skin, sinuses, kidneys, bladder, or prostate. Levofloxacin has been reported to be effective in eradicating Escherichia coli, a major pathogen causing urinary tract infections (McGregor et al., 2009).
Population-based compartment modeling has been developed since the 1970s until now. One of the advantages of the population-based compartment modeling approach is the focus of analysis on the whole population instead of the conventional method based on the two-stage approach based on the parameter values in each subject. The benefit of this condition is the completeness of information retrieval related to the absorption, elimination and/or distribution kinetics is contributed by the whole population (Mold and Upton, 2012; Sheiner et al., 1972).

Compartmental modeling uses the assumption that changes in the magnitude of a variable (e.g., concentration or amount of mass to 
time) are due to the movement of matter from one area to another. The compartment is defined as the material characteristics that can be in the form of a specific chemical form, biological material (organs, parts of organs) occupying a particular room or volume (Wastney, 1999).

The pharmacokinetics of levofloxacin have been examined in several reports (Cheng et al., 2002; Furlanut et al., 2003; Ghimire et al., 2016). Some of them also implemented the populationbased approach to estimate the model parameters. However, none of those studies was performed to find the best model based on a comparison of several approaches. In the present study, we focused on the method using Monolix, one of the alternative tools in population-based analyses (Chan et al., 2011; Dartois et al., 2007). We also performed pharmacokinetic studies in rabbits, considering the practicality and ease of handling the laboratory animals in blood sample collections.

\section{MATERIALS AND METHODS}

Levofloxacin hydrochloride (pharmaceutical grade) was purchased from PT. Kimia Farma Tbk (Bandung, Indonesia), whereas levofloxacin and ciprofloxacin (analytical grade) from SigmaAldrich (Buchs, Switzerland). Potassium dihydrogen phosphate, glacial acetic acid, phosphoric acid, acetonitrile, and methanol were of analytical grade and purchased from Merck (Darmstadt, Germany).

\section{Method}

Preparation of intravenous bolus and peroral levofloxacin solutions.

Levofloxacin solution was prepared at a concentration of $30 \mathrm{mg} / \mathrm{mL}$ in $0.9 \% \mathrm{NaCl}$. The mixture was vortexed for $1 \mathrm{~min}$ and sonicated for $5 \mathrm{~min}$ to obtain a homogeneous solution. The injection volume for intravenous bolus and peroral delivery in the rabbit was $1.5 \mathrm{~mL}$ and $2 \mathrm{~mL}$, respectively.

Pharmacokinetic studies of levofloxacin in rabbits. Six New Zealand white rabbits (the average weight of $2.9 \pm 0.5 \mathrm{~kg}$ ) were divided into two groups. The first group was received $45 \mathrm{mg}$ levofloxacin dose as an intravenous bolus injection in the first week, followed by $60 \mathrm{mg}$ of levofloxacin via a peroral route in the second week, and vice versa for the second group. Blood samples $(1 \mathrm{~mL})$ were taken from the rabbit ear vein at $0 \mathrm{~min} ; 10 ; 20 ; 30 ; 60$; $120 ; 180 ; 300$; and 480 after treatment and placed into Eppendorf tubes containing heparin. Samples were centrifuged to separate plasma. Plasma samples were then stored frozen $\left(-20^{\circ} \mathrm{C}\right)$ until analysis. The study protocols were approved by the Laboratory Animal Ethics Commission of the Integrated Research and Testing Laboratory, Universitas Gadjah Mada, Yogyakarta, Indonesia.

\section{Levofloxacin analysis}

A total of $300 \mu \mathrm{L}$ plasma was put into $1.5 \mathrm{~mL}$ Eppendorf tubes, followed by the addition of $50 \mu \mathrm{L}$ of $20 \mathrm{ppm}$ standard ciprofloxacin and $800 \mu \mathrm{L}$ acetonitrile. The mixtures were then centrifuged at $5,00 \mathrm{rpm}$ for $10 \mathrm{~min}$ at $4^{\circ} \mathrm{C}$. The supernatant was taken prior to filling into the vial. A total of $800 \mu \mathrm{L}$ acetonitrile was added back to the sludge, and the extraction process was repeated. This later supernatant was mixed with the first one, followed by a solvent evaporation step. Residues were stored at $-20^{\circ} \mathrm{C}$ until analysis. On the day of analysis, the residue was added with $1 \mathrm{~mL}$ of the mobile phase followed by a mechanical shaking for 1 min. Before injection into the HPLC (injection volume $20 \mu \mathrm{L}$ ), the solution was filtered using a $0.45 \mu \mathrm{m}$ membrane filter.

\section{Liquid chromatographic system}

Levofloxacin analysis was performed by using HPLC L-2000 Hitachi equipped with L-2130 pump, L-2200 autosampler, L2420 UV-Vis detector controlled by D-2000 HSM Elite software with a stationary phase of LiCrosphore ${ }^{\circledR}$ C18 column (length $250 \mathrm{~mm}$; i.d. $4.6 \mathrm{~mm}$; particle size $5 \mu \mathrm{m}$ ) and a mobile phase consisting of acetonitrile: methanol: $25 \mathrm{mM}$ pH 3.0 phosphate buffer (13: 7: 80), pumped at a flow rate of $1.5 \mathrm{~mL} / \mathrm{min}$. Levofloxacin detection was performed using an ultraviolet detector at a wavelength of $280 \mathrm{~nm}$.

\section{Data analysis}

Monolix (Frame, 2006), stand-alone version 2019R2 and running under Windows 10 machine, was used to analyze the data. Firstly, we implemented a population model with one compartment disposition model, followed by the same model but with a two-compartment model. Analyses were performed using the available template models provided by Monolix. The structural model of intravenous bolus injection with the one-compartment disposition model has the elimination rate constant $(\mathrm{K})$ and the distribution volume (Vd) parameters.

The model of peroral administration with the one-compartment model has the absorption rate constant $(\mathrm{Ka}), \mathrm{K}$, and $\mathrm{Vd}$ parameters. 

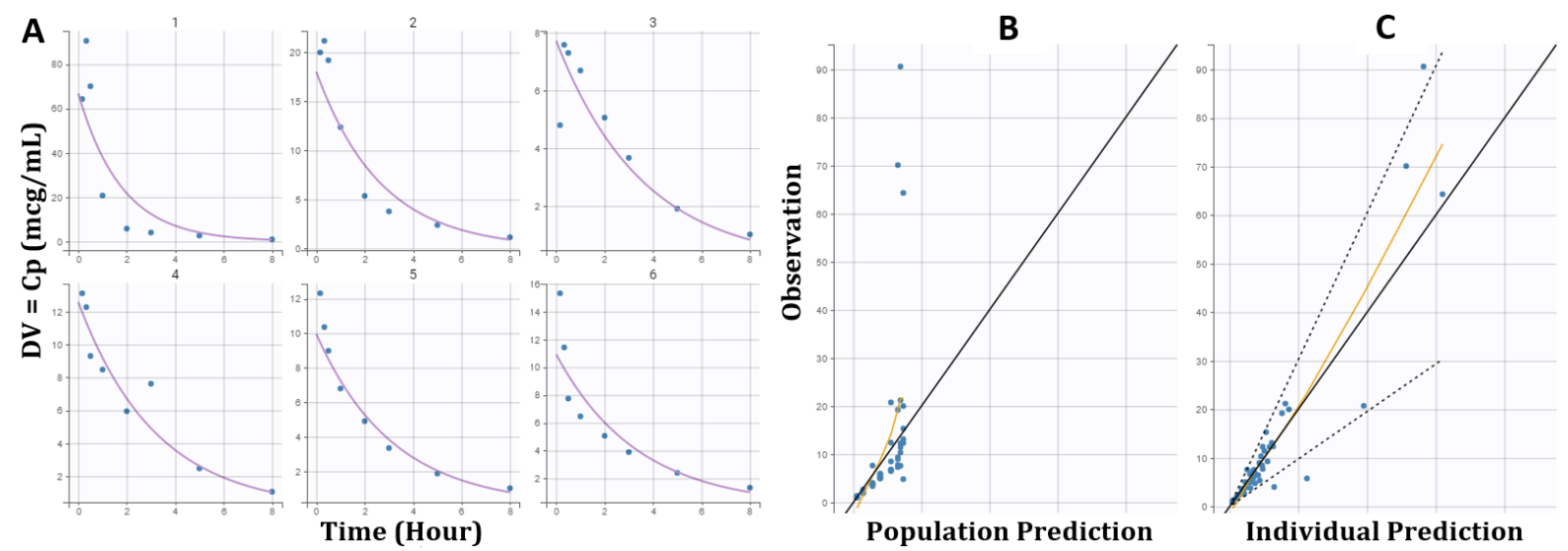

Figure 1. The goodness of fit of the one-compartment open model of levofloxacin after intravenous bolus injection, i.e., individual data fitting analyses (panel A); observation versus population prediction analysis (panel B) and observation versus individual prediction analysis (panel C).
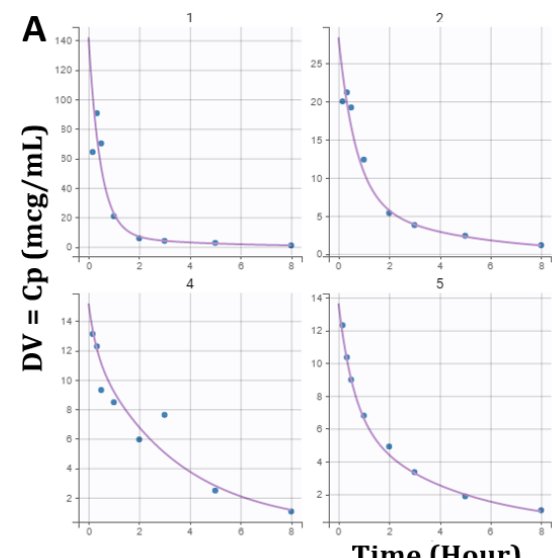

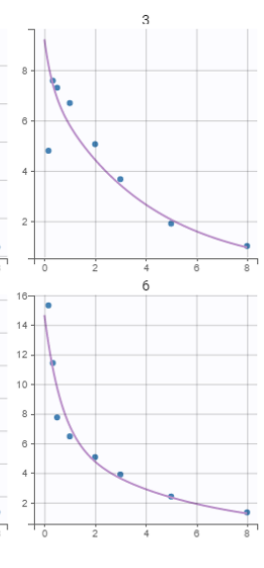

B

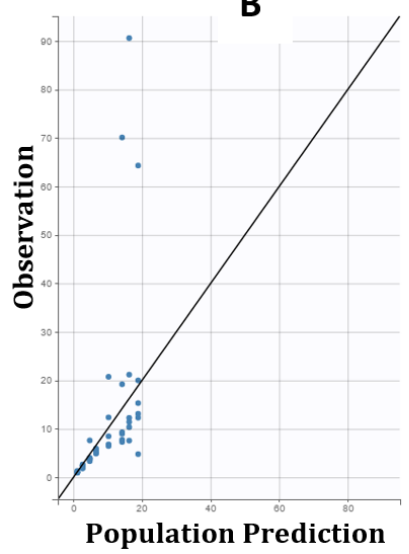

C

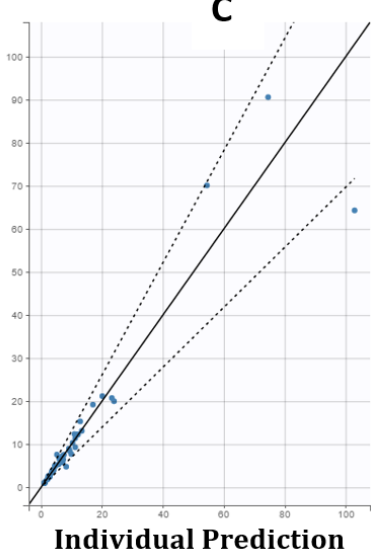

Figure 2. The goodness of fit of a two-compartment open model of levofloxacin after intravenous bolus injection, i.e., individual data fitting analyses (panel A); observation versus population prediction analysis (panel B) and observation versus individual prediction analysis (panel C), with a combination error model implemented.

The structural model of intravenous bolus injection with the two-compartment model has a distribution to the peripheral rate constant $\left(\mathrm{K}_{12}\right)$, the distribution to the plasma rate constant $\left(\mathrm{K}_{21}\right)$, $\mathrm{K}$, and $\mathrm{Vd}$ parameters. The model for peroral administration with the two-compartment model has the $\mathrm{Ka}, \mathrm{K}_{12}, \mathrm{~K}_{21}, \mathrm{~K}$, and $\mathrm{Vd}$ parameters. No covariate was applied while the covariance implemented the default diagonal pattern.

The adequacy of modeling in all cases was analyzed based on the goodness-of-fit evaluations. These evaluations consisted of 1) the individual fitting with the individual and population model prediction curves; 2) the correlation of DV, namely the dependent variable (the observed $\mathrm{Cp}$ ) versus population model prediction of $\mathrm{Cp}$; and 3 ) the correlation of DV versus the individual model prediction of $\mathrm{Cp}$. Such evaluations are considered crucial to judge the adequacy of specific modeling analyses (Mohammed et al., 2012; Owen and Fiedler-Kelly, 2014; Zheng et al., 2014).

\section{RESULTS AND DISCUSSION}

Analyses of pharmacokinetic profiles after intravenous bolus injection

Population-based analyses of levofloxacin administration via a bolus injection (Figure 1 (onecompartment open model) and Figure 2 (twocompartment open model)). As demonstrated in those two figures, it can be concluded that the population model with a two-compartment open model can better describe the data. 

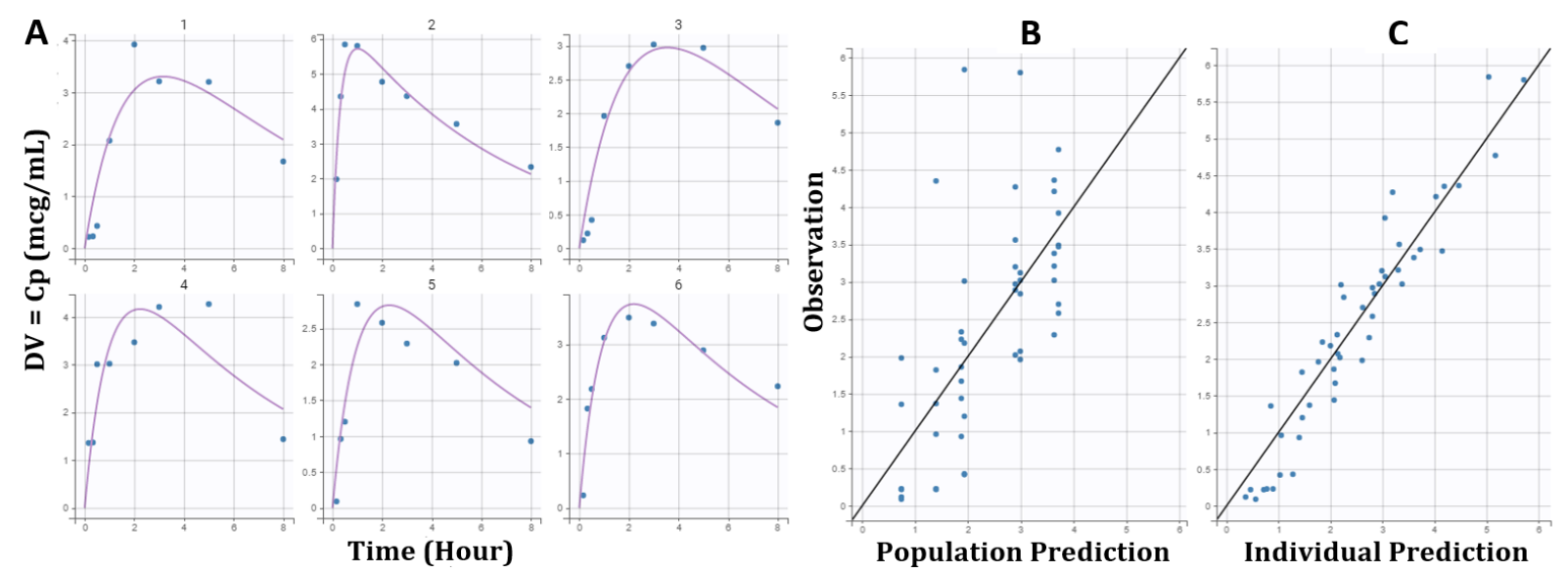

Figure 3. The goodness of fit of the one-compartment open model of levofloxacin after peroral administration, i.e., individual data fitting analyses (panel A); observation versus population prediction analysis (panel B), and observation versus individual prediction analysis (panel C).
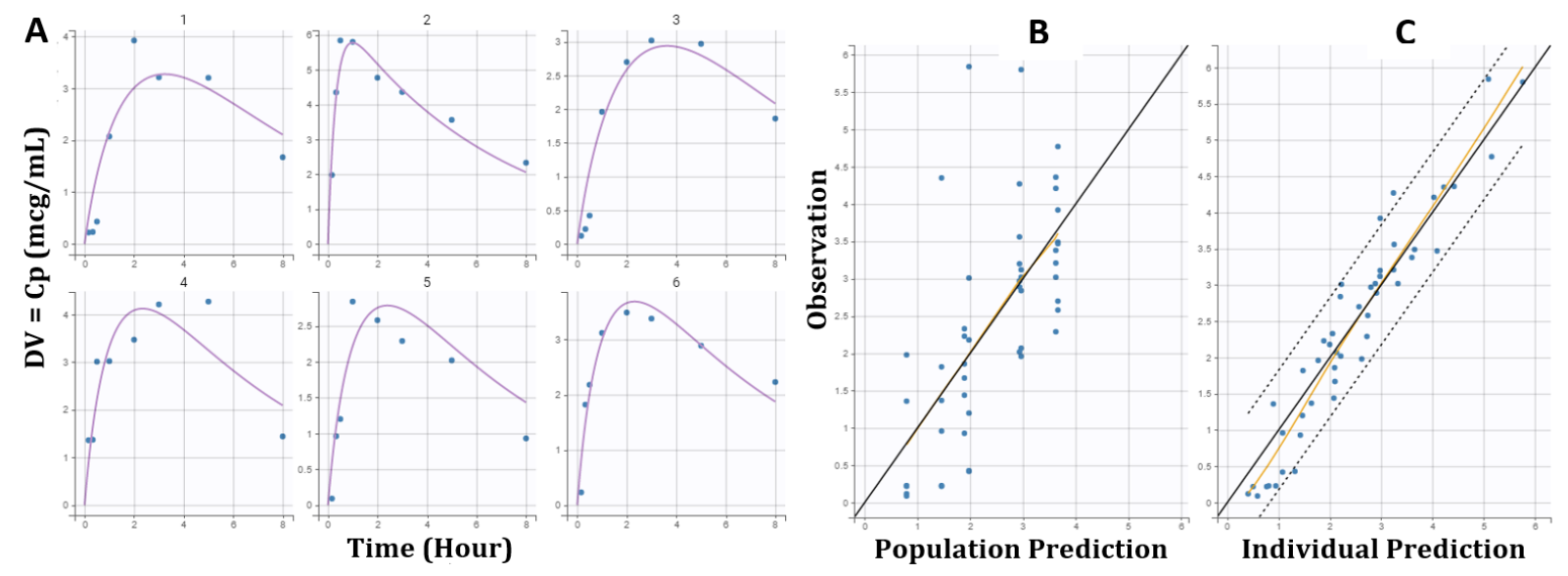

Figure 4. The goodness of fit of the two-compartment open model of levofloxacin after peroral administration, i.e., individual data fitting analyses (panel A); observation versus population prediction analysis (panel B) and observation versus individual prediction analysis (panel C), with a combination error model implemented.

It is clearly shown that the one-compartment model is unable to describe some points of the $\mathrm{Cp}$ versus time data.

Analyses of pharmacokinetic profiles following peroral administration

Population-based analyses of levofloxacin administration via peroral administration (Figure 3 and Figure 4) by one- and two-compartment open models, respectively.

Simultaneous fitting of intravenous bolus and peroral administration

Although the fitting with the twocompartment model has described well pharmacokinetic data of intravenous bolus and peroral administration, there is a lack of model parameters estimation. Inconsistency of the disposition parameter estimates following both routes of administration (Table I). In order to overcome this, simultaneous analysis of the data by combining all data in one modeling analysis was performed (Figure 5).

As can be seen from the figures, the simultaneous fitting provides better goodness of fit whilemaintaining thedisposition values parameters in each subject is the same (Table II). Moreover, the individual estimated values of $\mathrm{K}$ and $\mathrm{Vd}$ were relatively close to the previously published data of levofloxacin pharmacokinetics in rabbits. 

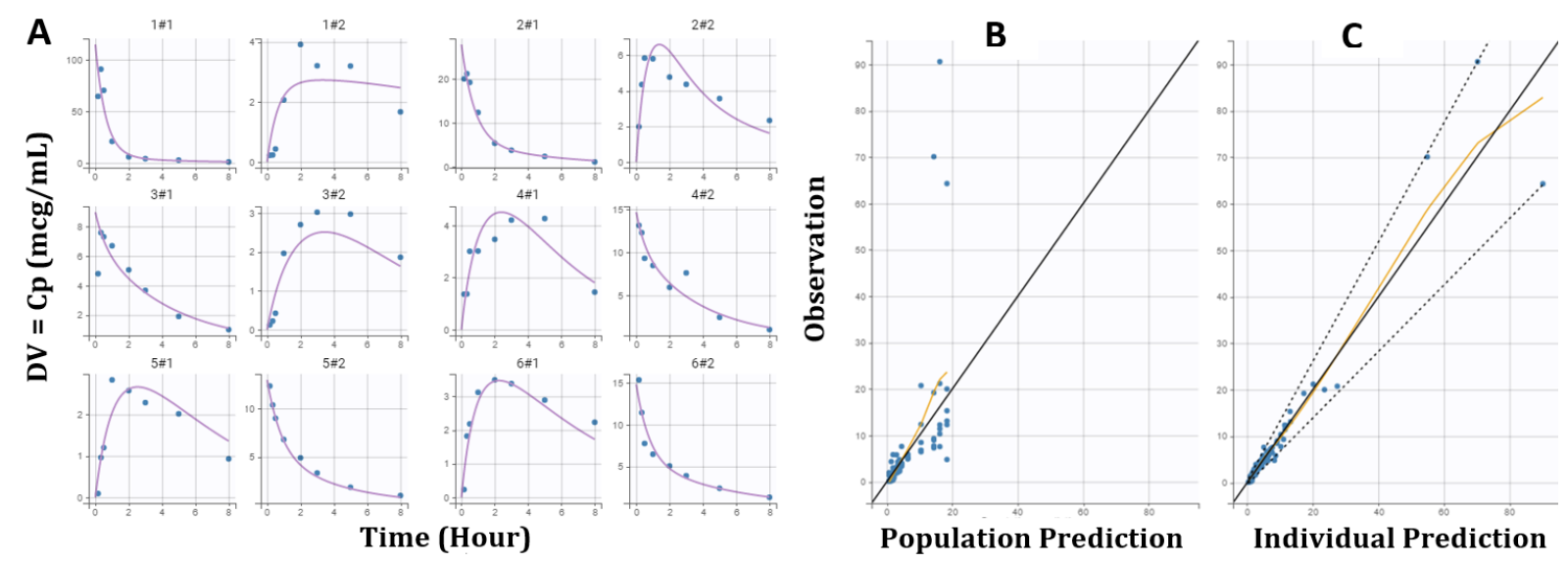

Figure 5. The goodness of fit of a two-compartment open model after a simultaneous fitting of intravenous bolus injection and peroral administration of levofloxacin, i.e., individual data fitting analyses (panel A); observation versus population prediction analysis (panel B) and observation versus individual prediction analysis (panel C), with a combination error model implemented.

Table I. The estimated individual values of levofloxacin pharmacokinetic parameters following intravenous bolus injection and peroral administrations.

\begin{tabular}{|c|c|c|c|c|c|c|c|c|c|c|}
\hline \multirow{2}{*}{ ID } & \multicolumn{2}{|c|}{ Vd (L) } & \multicolumn{2}{|c|}{$\mathrm{K}\left(\mathbf{h}^{-1}\right)$} & \multicolumn{2}{|c|}{$K_{12}\left(h^{-1}\right)$} & \multicolumn{2}{|c|}{$K_{21}\left(h^{-1}\right)$} & \multicolumn{2}{|c|}{ Ka $\left(h^{-1}\right)$} \\
\hline & IV & ORAL & IV & ORAL & IV & ORAL & IV & ORAL & IV & ORAL \\
\hline 1 & 0.324 & 8.66 & 1.45 & 0.201 & 0.515 & 0.562 & 0.358 & 2.43 & NA & 0.505 \\
\hline 2 & 1.59 & 7.03 & 0.583 & 0.197 & 0.514 & 0.58 & 0.496 & 2.25 & NA & 2.48 \\
\hline 3 & 4.88 & 8.94 & 0.308 & 0.201 & 0.518 & 0.578 & 3.45 & 2.35 & NA & 0.419 \\
\hline 4 & 2.99 & 7.89 & 0.348 & 0.199 & 0.517 & 0.573 & 3.64 & 2.37 & NA & 0.844 \\
\hline 5 & 3.3 & 11.33 & 0.415 & 0.205 & 0.519 & 0.586 & 1.04 & 2.23 & NA & 0.802 \\
\hline 6 & 3.06 & 8.71 & 0.377 & 0.199 & 0.526 & 0.588 & 0.828 & 2.24 & NA & 0.842 \\
\hline
\end{tabular}

Legend: NA: not available

Table II. The estimated parameters values of levofloxacin pharmacokinetics after a simultaneous analysis of intravenous bolus injection and peroral administration data.

\begin{tabular}{|c|c|c|c|c|c|c|}
\hline ID & f & $\mathrm{Ka}\left(\mathrm{h}^{-1}\right)$ & Vd (L) & $K\left(h^{-1}\right)$ & $K_{12}\left(h^{-1}\right)$ & $K_{21}\left(h^{-1}\right)$ \\
\hline 1 & 0.588 & 0.048 & 0.397 & 1.14 & 0.378 & 0.274 \\
\hline 2 & 0.593 & 0.665 & 1.65 & 0.513 & 0.493 & 0.383 \\
\hline 3 & 0.595 & 0.328 & 5.03 & 0.275 & 0.303 & 1.7 \\
\hline 4 & 0.587 & 0.542 & 3.1 & 0.331 & 0.357 & 1.63 \\
\hline 5 & 0.584 & 0.348 & 3.52 & 0.412 & 0.36 & 0.726 \\
\hline 6 & 0.591 & 0.449 & 3.07 & 0.354 & 0.49 & 0.65 \\
\hline Mean* & 0.59 & 0.397 & 2.795 & 0.504 & 0.397 & 0.894 \\
\hline $\mathrm{SD}^{* *}$ & 0.004 & 0.212 & 1.597 & 0.322 & 0.077 & 0.62 \\
\hline POP Value ${ }^{\#}$ & 0.588 & 0.304 & 2.16 & 0.439 & 0.354 & 0.575 \\
\hline Omega\#\# & 0.0425 & 0.875 & 0.836 & 0.498 & 0.474 & 0.86 \\
\hline$a^{* * \#}$ & \multicolumn{6}{|c|}{0.249} \\
\hline $\mathrm{b}^{\# * *}$ & \multicolumn{6}{|c|}{0.173} \\
\hline
\end{tabular}

\#\# the standard deviation of the interindividual variability of pharmacokinetic parameters estimated using Monolix; **\# the proportional error estimated using Monolix; \#\#* the additive error estimated using Monolix 
standard deviation of the interindividual variability of $\mathrm{Vd}$ and $\mathrm{K}$ at $2.16 \mathrm{~L}$ and $0.439 \mathrm{~h}^{-1}$, as well as 0.836 and 0.498 , respectively (Table 2 ).

We calculated using Monolix the approximate values of $\mathrm{Vd}$ and $\mathrm{K}$ from the mean plasma concentration of levofloxacin time profiles data reported by Sitovs et al. (2020) at a value of $2.35 \mathrm{~L}$ and $1.04 \mathrm{~h}^{-1}$, respectively. Those parameters are reasonably close to the arithmetic mean \pm standard deviation of the individual $\mathrm{Vd}$ and $\mathrm{K}$ at $2.795 \pm 1.597 \mathrm{~L}$ and $0.504 \pm 0.322 \mathrm{~h}^{-1}$. (Table 2). These facts suggest a proper simultaneous population modeling of levofloxacin plasma concentrations data.

\section{CONCLUSION}

Levofloxacin plasma concentration-time profiles in rabbits delivered by intravenous bolus injection and peroral administration can be described adequately by the population model using a two-compartment open model. The estimated disposition parameter models in each rabbit are different depending on the route of drug administration. Simultaneous fitting demonstrates the adequacy of fitting in all subjects.

\section{ACKNOWLEDGEMENT}

The present research was supported by Hibah Penelitian Dasar Unggulan Perguruan Tinggi, a research grant scheme provided by the Ministry of Research, Technology and Higher Education, the Republic of Indonesia, the year 2018-2019.

\section{REFERENCES}

Chan, P.L.S., Jacqmin, P., Lavielle, M., McFadyen, L., dan Weatherley, B., 2011. The use of the SAEM algorithm in MONOLIX software for estimation of population pharmacokinetic pharmacodynamic-viral dynamics parameters of maraviroc in asymptomatic HIV subjects. J. Pharmacokinet. Pharmacodyn. 38: 41-61.

Cheng, F.C., Tsai, T.R., Chen, Y.F., Hung, L.C., Tsai, T.H., 2002. Pharmacokinetic study of levofloxacin in rat blood and bile by microdialysis and high-performance liquid chromatography. J. Chromatogr. A. 961, 131-136.

Dartois, C., Lemenuel-Diot, A., Laveille, C., Tranchand, B., Tod, M., dan Girard, P., 2007.Evaluation of uncertainty parameters estimated by different population PK software and methods. J. Pharmacokinet. Pharmacodyn. 34: 289-
311.

Fu, K.P., Lafredo, S.C., Foleno, B., Isaacson, D.M., Barrett, J.F., Tobia, A.J., dkk., 1992. In vitro and in vivo antibacterial activities of levofloxacin (1-ofloxacin), an optically active ofloxacin. Antimicrob. Agents Chemother. 36: 860-866.

Furlanut, M., Brollo, L., Lugatti, E., Di Qual, E., Dolcet, F., Talmassons, G., Pea, F., 2003. Pharmacokinetic aspects of levofloxacin $500 \mathrm{mg}$ once daily during sequential intravenous/oral therapy in patients with lower respiratory tract infections. J. Antimicrob. Chemother. 51, 101-106.

Ghimire, S., Van't Boveneind-Vrubleuskaya, N., Akkerman, O.W., de Lange, W.C.M., van Soolingen, D., Kosterink, J.G.W., van der Werf, T.S., Wilffert, B., Touw, D.J., Alffenaar, J.-W.C., 2016. Pharmacokinetic/pharmacodynamic-

based optimization of levofloxacin administration in the treatment of MDRTB. J. Antimicrob. Chemother. 71, 26912703.

McGregor, J.C., Allen, G.P., Bearden, D.T., 2008. Levofloxacin in the treatment of complicated urinary tract infections and acute pyelonephritis. Ther. Clin. Risk Manag. 4, 843-853.

Mohammed, B.S., Engelhardt, T., Cameron, G.A., Cameron, L., Hawksworth, G.M., Hawwa, A.F., McElnay, J., Helms, P.J., McLay, J.S., 2012. Population pharmacokinetics of single-dose intravenous paracetamol in children. Br. J. Anaesth. 108, 823-829.

Mould, D.R. dan Upton, R.N., 2012. Basic Concepts in Population Modeling, Simulation, and Model-Based Drug Development. CPT: Pharmacometrics Sys. Pharmacol. 1: e6, https://ascpt.onlinelibrary.wiley.com/doi /10.1038/psp.2012.4

Owen, J.S., Fiedler-Kelly, J., 2014. Introduction to population

pharmacokinetic/pharmacodynamic analysis with nonlinear mixed effects models. Wiley, Hoboken, New Jersey.

Sheiner, L.B., Rosenberg, B., Melmon, K.L., 1972. Modelling of individual pharmacokinetics for computer-aided drug dosage. Comput. Biomed. Res. 5, 411-459.

Sitovs, A., Voiko, L., Kustovs, D., Kovalcuka, L., Bandere, D., Purvina, S., Giorgi, M.. 2020. Pharmacokinetic profiles of levofloxacin 
after intravenous, intramuscular and subcutaneous administration to rabbits (Oryctolagus cuniculus). J. Vet. Sci. 21(2):e32,

https://www.vetsci.org/DOIx.php?id=10.41 42/jvs.2020.21.e32

Wastney, M.E., Patterson, B.H., Linares, O.A., Greif, P.C., Boston, R.C., 1998. Investigating Biological Systems Using Modeling:
Strategies and Software, Academic Press, San Diego.

Zheng, S., Matzneller, P., Zeitlinger, M., Schmidt, S., 2014. Development of a Population Pharmacokinetic Model Characterizing the Tissue Distribution of Azithromycin in Healthy Subjects. Antimicrob. Agents Chemother. 58, 6675-6684. 